\title{
Development of Coherent, Expandable, Reconfigurable Instrument Node (ERIN) for Web Sensor Applications
}

\author{
L. Hilliard \\ NASA Goddard Space Flight Center \\ Greenbelt, MD 20771 \\ 301-286-9294 \\ Lawrence.M.Hilliard@nasa.gov
}

\begin{abstract}
Microwave radars operating over the L-band microwave spectrum are often used as sensors for estimation of vegetation biomass, land surface topography and surface deformation, surface soil moisture, and polar ice sheets thickness. Monitoring of these parameters is essential for gauging the Earth's ecosystem. Because of restriction on the mass and size of a radar sensor that can be deployed on a small aircraft/spacecraft, the surface resolution with which these essential earth parameters can be improved using this technology by forming interferometric baselines between nodes. The Expandable Reconfigurable Instrument Node (ERIN) will provide a semi-closed loop system solution for a variety of sensors. The ERIN baselines a reconfigurable processing technology with required memory to allow on-board processing of science data. Standardized interfaces are provided to allow for interfacing to attitude control instrumentation such as Global Positioning Systems (GPS) and Inertial Measurement Units (IMU). A communications device will be added to the node that would allow for node-to-node communications. ${ }^{12}$
\end{abstract}

\section{TABLE OF CONTENTS}

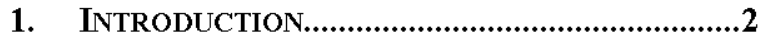

2. INSTRUMENT DESCRIPTION ..................................2

3. ERIN INTERFACES.............................................4

4. ERIN WEB SENSOR STRAND VERIFICATION...4

5. ERIN-WSS ALGORITHM.....................................5

6. ERIN-WSS USE CASES .......................................6

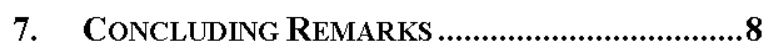

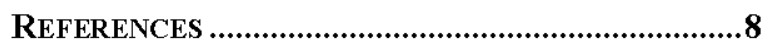

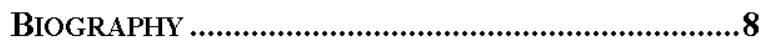

\footnotetext{
1

${ }^{1}$ U.S. Government work not protected by U.S. copyright

2 IEEEAC paper \#1324, Version 2, Updated January 9, 2008
} 


\section{INTRODUCTION}

\section{ERIN Background}

A requirement for a small, lightweight, reconfigurable instrument data system has been identified through collaborations with various instrumentors. The concept of ERIN, an Expandable, Reconfigurable Instrument Node, was produced to fit that need and to provide small individual sensors access to on-board processing that would not otherwise be possible. The following set of requirements was identified as being critical to the success of ERIN:

-FPGA-based therefore reconfigurable in-flight if needed

-Expandable - standardized interfaces will allow additional components to be added

Wireless communication

GPS

Inertial Measurement Unit

-Hardware specifications would meet requirements for a variety of instruments

-Algorithms could be changed to meet processing needs

-Low overhead for configuration of on-board devices

-Standard enough to use on multiple instrument platforms

The key requirement is to take advantage of the reconfigurability of FPGAs to fit a wide variety of small individual sensors. Position and pointing knowledge are key to meeting the web sensor strand application. Therefore the wireless communication, GPS, and IMU tags are optional but necessary for this most ambitious of applications.

\section{WSS Background}

Although the Web Sensor Strand (WSS) technique will only be shown for two nodes in this development program, it will be relevant for many nodes that need to act in coherence. This is a new capability that enables small platforms to act as a large sensor. It is limited to targets with radar returns that are not changing in time such as subterranean layers of material.

\author{
-Present technology \\ -Sensor webs utilize multiple individual sensors \\ -Each sensor acts individually \\ -No advantage taken of multiple sensor nodes in sensor \\ web
}

-Sensor web limited by spatial resolution and individual sensor sensitivity

-ERIN technology

-Multiple sensors configured in a web

-Sensors act in coherence

-Processing of data combined across multiple nodes

-Enhances spatial resolution and sensitivity

-Produces more meaningful scientific experiments

ERIN-WSS Goal

The ERIN-WSS goal is to enable relevant science measurements with microwave instruments (radars and radiometers) using small platforms to create a large transmitting as well as collecting aperture.

The phase lock loop maintained between nodes must remain in lock to form multiple baselines from a common wave front. Each baseline must be translated to a common coordinate system for image processing to work. If the phase difference between nodes drifts more than $1 / 4$ wavelength then the phase lock will not be robust. This can drift because of uncertainties in the absolute position of the antenna phase center in space (global positioning system or GPS), or the relative position (Inertial Measurement Unit - IMU measuring roll, pitch, or yaw) of the beam relative to the absolute position sensor.

\section{INSTRUMENT DESCRIPTION}

The ERIN monostatic sensor operates at $1.26 \mathrm{GHz}$ with a nominal pulse width of $30 \mathrm{nsec}$ (selectable). The instrument consists of a single channel (Fig 1). 


\section{ERIN Radar System Block Diagram}

$7 / 30 / 2007$

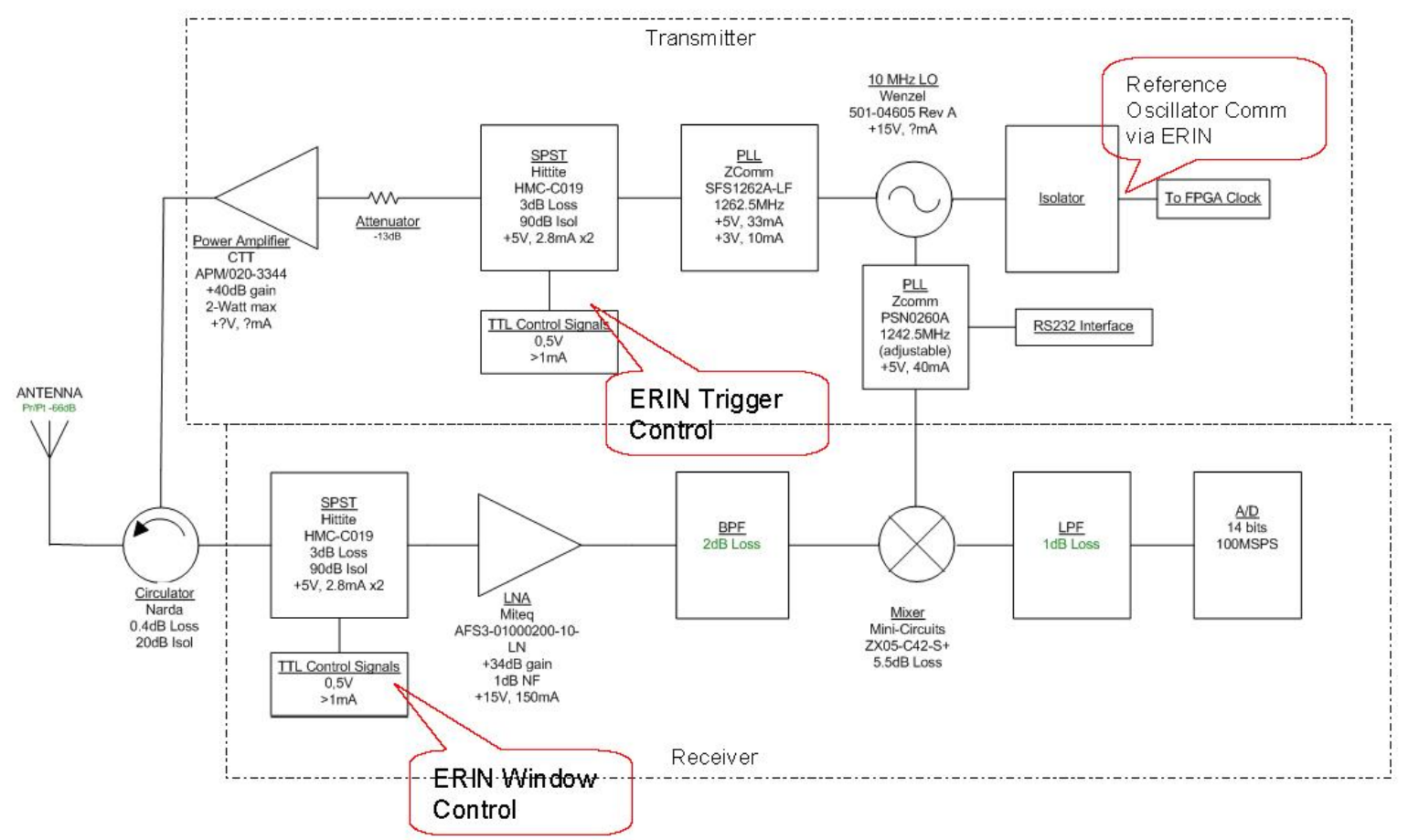

Figure 1 Architecture of an ERIN Transmit/Receive Module.

The transmit channels include solid state power amplifiers (SSPA), well isolated RF switches for triggering the transmit pulse and closing the return pulse window for the next pulse, and a reference oscillator for tieing two ERIN contrlled sensors together synchronously. The SSPAs generate up to only 2 Watts of total output power because the goal is to fly low and slow on small platforms[1]. The receive channels include low noise amplifiers, filters, and frequency down-converters.

The antenna is a coax fed microstrip patch-array made up of 3 U-slot elements that have flown in the wing of a Uninhabited Aerial Vehicle (UAV). The lightweight array was available from past research[5], meets the lightweight and wideband requirements of the terrestrial web sensor strand test. Table 1 summarizes the characteristics of the radar. The Expandable Reconfigurable Instrument Node is a compact, lightweight and re-configurable digital data processing system. ERIN is capable of aggregating data from a variety of sensor sources, processing sensor data at very high rates, and networking with other ERIN
TABLE I RADAR NODE CHARACTERISTICS

\begin{tabular}{|c|c|}
\hline Frequency & $1.26 \mathrm{GHz}$ \\
\hline PRF period & $2 \mathrm{~ms}$ \\
\hline Pulse Width & $30 \mathrm{~ns}$ \\
\hline Number of Transmitters & 1 \\
\hline Maximum Output Power & $2 \mathrm{~W}$ \\
\hline Intermediate Frequency & $20 \mathrm{MHz}$ \\
\hline Antenna Type & Microstrip Patch Array \\
\hline Number of Patches & 3 \\
\hline Number of Active Subarrays & 1 \\
\hline A/D Sampling rate & $100 \mathrm{MSPS}$ \\
\hline Subarray Gain & $\sim 6 \mathrm{~dB} @ 1.26 \mathrm{GHz}$ \\
\hline 3 dB Beamwidth (Nadir, along \\
track, one-way) & $40 \mathrm{Degrees}$ \\
\hline Side Lobes (one-way) & Better than $-17 \mathrm{~dB}$ \\
\hline ERIN Dimensions & $\sim 6$ "X6"X3" \\
\hline Estimated Total System Power & $10 \mathrm{~W}$ \\
\hline Estimated Total System Weight & $3 \mathrm{Lbs}$ \\
\hline
\end{tabular}


systems forming a highly reconfigurable, flexible and coherent sensor network. The core of ERIN is based on a Xilinx Virtex-4 FPGA that has an embedded PowerPC 403 processor.

\section{ERIN INTERFACES}

- 50-pin General purpose I/O header that connects the processor directly to any custom sensor interface, or any standard digital communications interface such as PCI, FireWire, CameraLink, SPI and I2C.

- RS-232 Interface for connecting devices such as GPS, Inertial Measurement Units (IMU) and temperature sensors.

- $100 \mathrm{Mbps}$ Ethernet for connecting an ERIN node to a host PC for data storage and archival.

- Wireless Network Communications Interface for wireless interoperability between a family of ERIN nodes, allowing for the creation of a dynamic expandable network of sensors. This provides the capability for doing applications such as interferometric SAR, differential GPS, bistatic Radar for example.

\section{ERIN WEb SENSOR STRAND VERIFICATION}

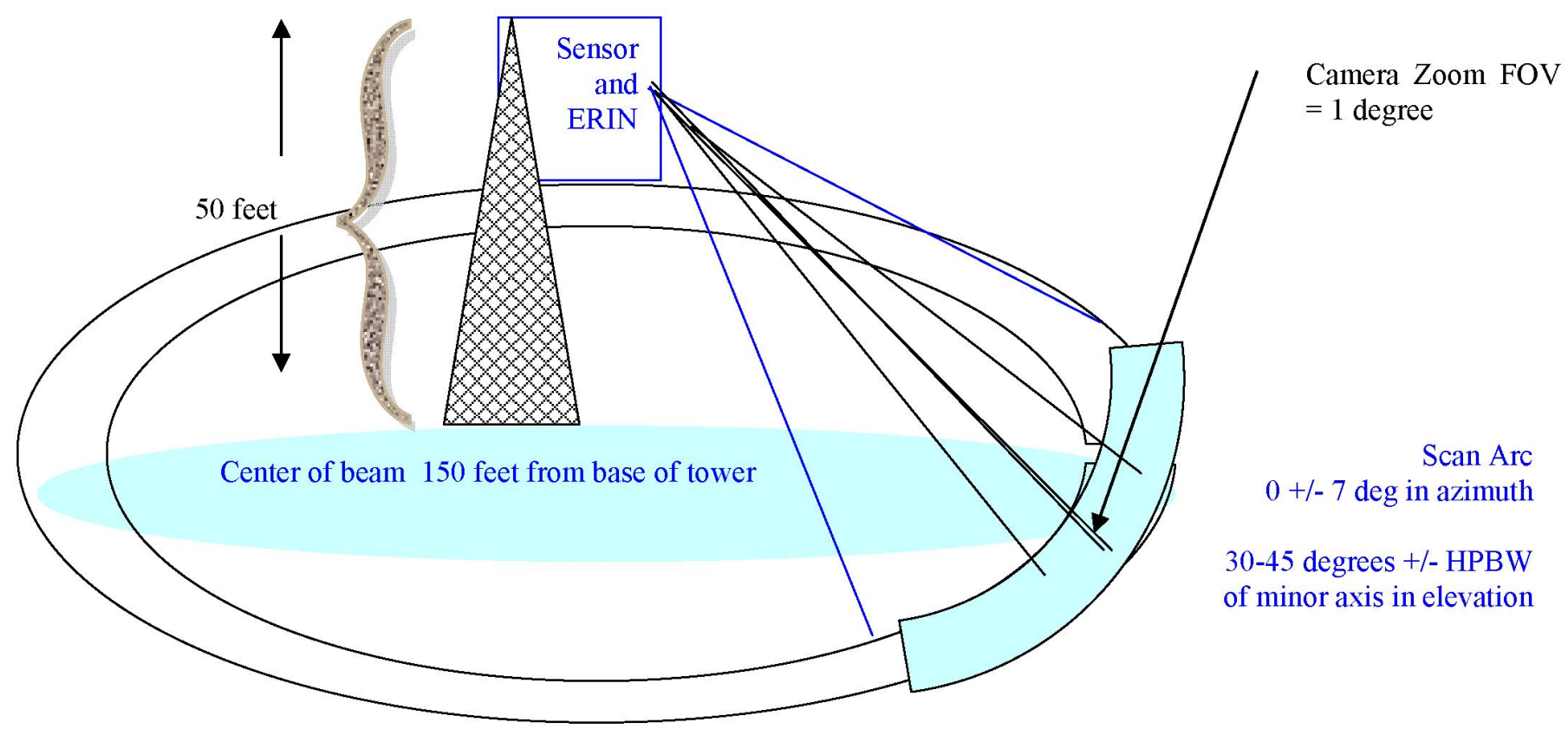

Figure 2 The Outdoor Monostatic Test for ERIN Radar characterization 
The Anechoic Chamber Test demonstration of the monostatic radar status was imminent in December 2007[6]. The radar (sensor) brassboard and U-slot ERIN antenna and prototype ERIN were integrated in weatherproof boxes for testing on the lab bench with a delay line and 2-way propogation in the anechoic chamber under ERIN breadboard trigger control. From there 2-way (transmit/receive) pulse analysis for delay, spreading, dynamic range and signal to noise characterization will be done. RF data storage in bench test equipment (BTE) such as a digital oscilloscope. For the interim, replay from the scope through a Graphical User Interface(GUI) will enable and help troubleshoot the capability to have a quick-look at verifying and validating data.

In Figure 2, the 50 foot tower test configuration is described that will test ERIN in a "relevant" noise environment. The monostatic radar will need to be extremely fast to gate out the clutter from surrounding reflective sources (e.g. buildings and fences). The tower configuration requires that ERIN store and tag the data and temperature telemetry and return it to the operator via a serial cable (RS-232). An ethernet core will upgrade the data extraction capability in subsequent versions of ERIN.

Tagged data storage and ingest to the image processing computer will be pursued in parallel in the ERIN development laboratory using the 6" $\times$ 6" ERDN-Lite breadboard. When the real-time troubleshooting of the front end with the scope, and the real-time algorithm integration and testing on the ERIN-Lite (including the Ethernet controller algorithm and packetizer) is complete, then the monostatic radar system will be tested end-to-end.

\section{ERIN-WSS ALgORITHM}

ERIN-WSS Algorithm The flow chart, Figure 3, illustrates how the ERIN Web Sensor Strand is collected, tagged, buffered, enabling the image processing as work to be addressed in years 2 and 3 of the ERIN-WSS program.
First, the ERIN triggers and tags a radar pulse in the transmitting sensor (radar).

Second, the pulse, in our model is represented in the frequency domain as a carrier frequency surrounded by modulation

Third, staying in the frequency domain we have modeled the propagating to the far field through the test antenna (step 3) to the target and reflected scatter back to the source node and eventually other nodes in the model (step 4).

The reciprocal process (step 5), the antenna receives the returning pulse and it becomes important to return to a time-domain representation

In step 6, ERIN gating opens the receiver to accept the scattered energy.

To enable the image processing computer to do Multibaseline image processing - (multiple pulses, multiple node locations), ERIN must tag and buffer the return pulses.

ERIN triggers and tags the next pulse at the pulse repetition frequency (PRF) programmed by the user.

In the image processing computer, multiple baseline image processing algorithms will reside that take the GPS "time and position" tags and the azimuth and elevation "beam pointing" tags and reconstruct an array of multiple pulse returns from multiple node locations.

In the monostatic demonstration the position doesn't really change but it does establish a stationary node calibration of expected delay, dynamic range, and signal to noise. This calibration will be useful in processing node 2 returns when the node1-node 2 communication is established for the web sensor strand.

A predictive model for the expected return at multiple baselines is being developed by the team. The monostatic response was predicted for the short range of the 30 foot anechoic chamber and 71 foot slant range.[7] 


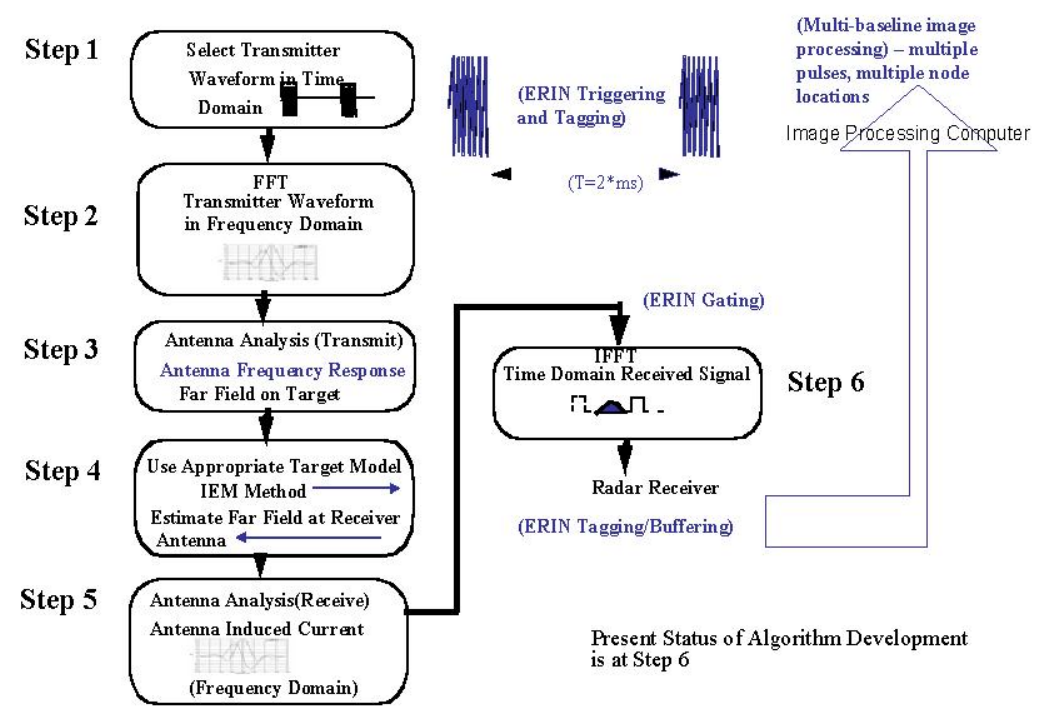

Figure 3 The ERIN Node is a synchronizer, pulse trigger controller, and tags radar return data with time, location, and platform orientation

\section{ERIN-WSS USE CASES}

\section{ERIN as a Node in a Sensor Web}

Every 2 milliseconds ERIN is triggering another snapshot of radar backscatter and buffering a precisely time and geolocation tagged beam. Though the L-Band ERIN Antenna was optimized for the radiometer frequency, the wideband antenna also works well at the nearby radar frequency. Both frequencies are used for Aquarius (currently under NASA development) and SMAP ( a decadal survey mission concept for soil moisture and freeze-thaw for weather and water cycle processes).
Multi-probe is a UAV mission that will take advantage of the lightweight ERIN and the equally lightweight Directdetect radiometer for the Wing of a UAV that flew in June 2007. In figures 4 and 5 there are examples of tracking LBand brightness temperature for each integration period. ERIN automatically records the data strand by strand. Coprocessing active and passive microwave data (and that of other sensors) on Multiprobe should be easily enabled by ERIN.

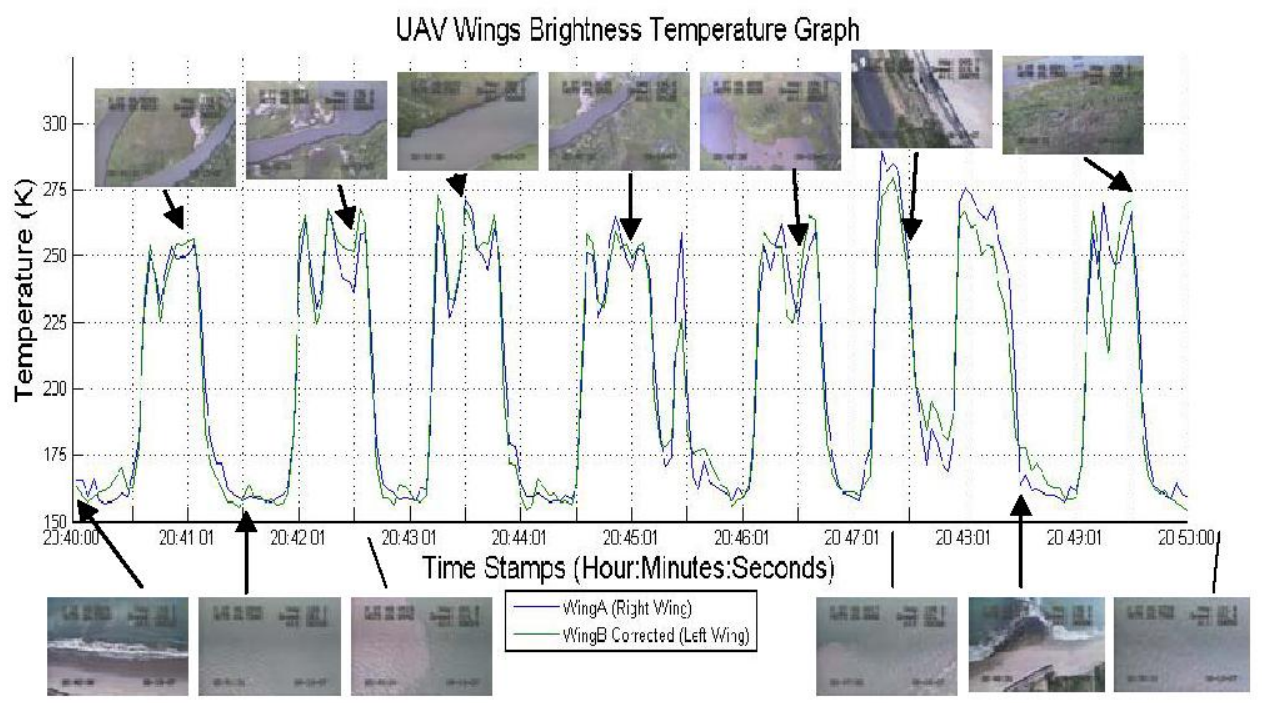


Figure 4 June 2007 Brightness Temperature Plot from Aerotenna UAV. Same as Multiprobe Application of ERIN (L-Band Radiometer), a data system capable of active/passive, and as a microwave node in a sensor web

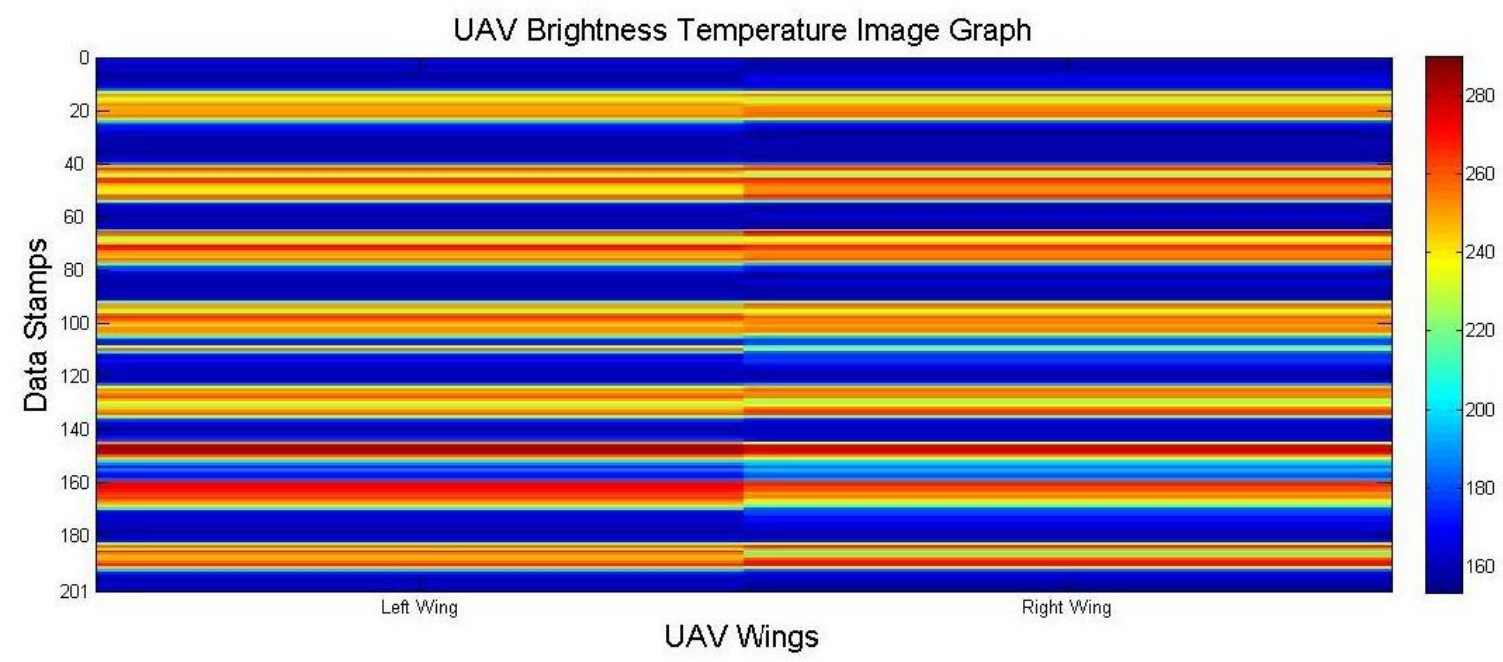

Figure 5. Strip map of brightness temperatures from 6/18/07 Aerotenna UAV

\section{ERIN planned as Web Sensor}

Precise recording of radar returns interleaved with verifiable snow water equivalent (SWE) brightness temperature data shows promise as the finest spatial resolution achievable to date using active-passive processing together. The science and technology development team of Slow and Low UAV for Snow Hydrology (SLUSH) has proposed this Instrument Incubator Probe. The SLUSH instruments will be using the reprogrammability of ERIN to create a Synthetically Thinned Aperture Radiometer[2] (STAR) correlator and interleave integration periods with radar returns of similar wavelengths. Active/Passive arrays at X-band, K-band, and Ka Band will be enabled to compare brightness temperature and synthetic array radar returns, strand by strand, and verify these with snow depth and wetness readings sampled by ground truth. The data shown in figure 5 drawn from the Cold Land Processes Experiment (CLPX) in 2003.

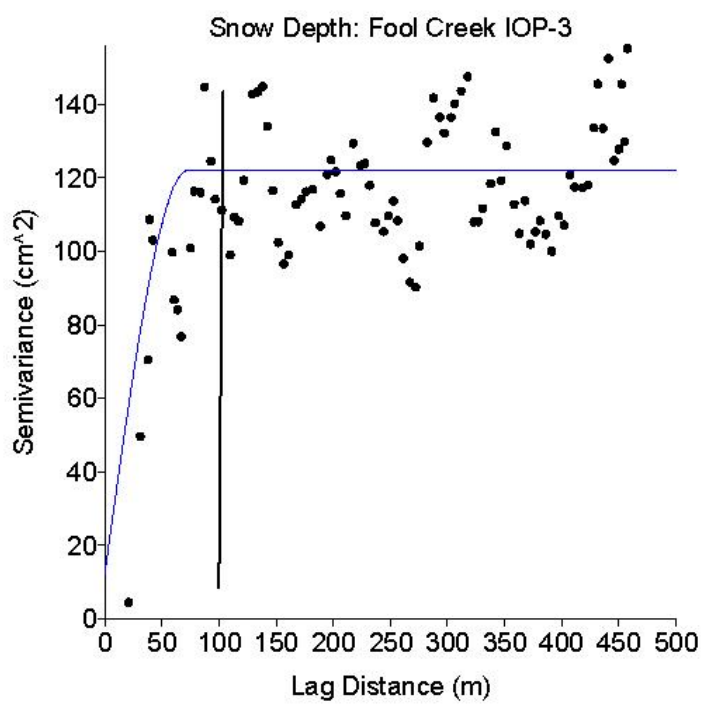

Figure 6. Snow Depth uncertainty due to inhomogeneity (50-100 meter correlation threshold $)^{11}$ Data Contributed by Dr. Richard Kelly Cold Land Processes Working Group Experiments 2003 (CLPXI)

RadSTAR combined L-Band Active (RadSTAR-A aka LIS) and Passive (RadSTAR-P aka ESTAR) instruments in a common flight with common beams in January and February 2007[3][4] to collect just this type of active/passive data, however the data was not collected synchronously in a common data system such as ERIN so image comparisons cannot be synthesized together.

A tie-in to the global hydrology cycle is a UAV mission named Synthetic Thinned Interferometer for Tomographic Cryospheric Hydrology (STITCH) mission. 
When ERIN reaches its full TRL advancement in 2009 , STITCH is being considered so as to provide a high resolution coastal survey of the Greenland Ice Sheet and another UAV to circumnavigate Antarctica. The melt from the north and south poles coastal areas actually feeds the deepest water in the ocean.

\section{CONCLUding REMARKS}

The Web Sensor Strand technology demonstration and the Instrument Incubator Program applications show the reconfigureability of ERIN to perform as the radiometer node in a multi-instrument in a Sensor Web data system that can fly on a small UAV such as Multiprobe. Active-Passive data retrieval, once demonstrated with ERNN will be enabled by fast datataking and slow and low flying on the snow water equivalent (SWE) measuring mission SLUSH. This interleaved active and passive data, when processed together, can produce large synthetic apertures using small platforms. SLUSH will be an excellent example of the potential of a web sensor to collect high resolution verifiable data. he was a spaceflight instrument manager for most of his career dating back to 1983 .

\section{REFERENCES}

[1] Hilliard, Lawrence M., P. Hildebrand, T. Markus , C. Johnson, G. Bland, R. Lawrence, and N. Phelps "Microwave Instrumentation for UAV Platforms Enabling Thin Ice Measurement" Arlington VA, Sept. 2005

[2] Le Vine, D.M., "Synthetic Aperture Radiometer Systems", IEEE Trans. Geosci. Remote Sens., Vol. 47, 2228-2236, 1999.

[3] Rincon, R.F. , P. Hildebrand, L. Hilliard "RadSTAR L-Band Imaging Scatterometer: Performance Assessment", 2007 Intemational Geoscience and Remote Sensing Symposium (IGARSS), July 2007.

[4] Rincon, R. P. Hildebrand, L. Hilliard, and J. Mead, "NASA's L-Band Imaging Scatterometer," Proceedings of IGARSS 2004, Anchorage, Alaska, Sept. 2004.

[5] Hilliard, L., R. Rincon, P. Hildebrand,, and J. Mead, "Lightweight Linear Broadband Antennas enabling small UAV Wing Systems and Space flight nanosat Concept", Proceedings of IGARSS 2004, Anchorage, Alaska, Sept. 2004.

[6] Hestnes, P. , L. Hilliard, and M. Deshpande, "Quarterly Report Num: AIST-05-0039-T-JAN-2008", Developing an Expandable Reconfigurable Instrument Node as a Building Block for a Web Sensor Strand, NASA ESTO Program 2007-2009.

[7] Deshpande, M. "Validation of EM Simulation Procedure", from FY07 Annual Review, Developing an Expandable Reconfigurable Instrument Node as a Building Block for a Web Sensor Strand, NASA ESTO Program 2007-2009.

\section{BIOGRAPHY}

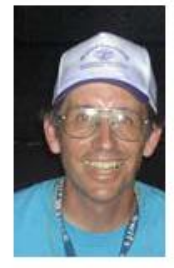

Larry Hilliard is an RF Systems Engineer at NASA Goddard Space Flight Center and has worked on various airborne microwave systems in the period 2002-2007. Before that 